\title{
Atendimentos realizados no Serviço de Traumatismos Dentários da FOP-Unicamp durante o período de dois anos
}

\section{Care provided at the Dental Trauma Service of the FOP-Unicamp over two years}

\author{
Andréa Cardoso Pereira* \\ Ana Carolina Correia Laurindo de Cerqueira Neto* \\ Thiago Farias Rocha Lima* \\ Alexandre Augusto Zaia*** \\ Adriana de Jesus Soares ${ }^{* * * *}$
}

\section{Resumo}

Objetivo: verificar a frequência dos atendimentos realizados no Serviço de Traumatismos Dentários da FOP-Unicamp, durante o período de fevereiro de 2013 a fevereiro de 2015. Sujeitos e método: os dados foram coletados na ficha clínica utilizada no serviço e foram avaliados os seguintes aspectos: gênero, faixa etária, classificação do dente, período entre o trauma e o primeiro atendimento no serviço, etiologia, classificação da injúria traumática e procedimento efetuado. Os dados foram analisados com auxílio do software SAS e do teste Qui-Quadrado, que foi utilizado para verificar a presença ou a ausência de significância com nível de 5\%. Resultados: foram analisadas as fichas clínicas de 57 pacientes com 161 dentes traumatizados, com maior prevalência do gênero masculino e da faixa etária abaixo dos 14 anos. Os incisivos centrais $(60,9 \%)$ foram os dentes mais afetados. A maioria dos pacientes $(56,1 \%)$ procurou atendimento entre 0 e 30 dias após o trauma. As quedas $(33,3 \%)$ foram a etiologia mais comum. As injúrias dentárias traumáticas mais encontradas foram as fraturas coronárias de esmalte e dentina (19,9\%) e a subluxação $(19,3 \%)$. O procedimento mais efetuado foi a realização dos exames clínico e radiográfico $(46,6 \%)$. Conclusão: os achados mais prevalentes dos atendi- mentos foram: o gênero masculino; a maior frequência de injúrias na faixa etária abaixo dos 14 anos de idade; os incisivos centrais como os dentes mais acometidos; o período entre 0 e 30 dias para procurar atendimento; as quedas, a etiologia mais comum; os exames clínico e radiográfico, o procedimento mais efetuado; as fraturas coronárias de esmalte e dentina e a subluxação, as injúrias mais observadas.

Palavras-chave: Endodontia. Epidemiologia. Traumatismos dentários.

\section{Introdução}

Os traumatismos dentários são frequentes e ocorrem, principalmente, em crianças, mas, também, são observados em adolescentes e adultos ${ }^{1}$. Atualmente, as injúrias dentárias traumáticas são encontradas em maior prevalência devido ao aumento da violência na sociedade, à grande quantidade de acidentes de tráfego e acidentes ocasionais envolvendo crianças e adolescentes por causa de

\footnotetext{
Mestra em Clínica Odontológica, área de Endodontia da FOP-Unicamp.

* Doutor em Clínica Odontológica, área de Endodontia da FOP-Unicamp. Professor da área de Endodontia da UFPB.

*** Doutor em Biologia e Patologia bucodental. Professor livre-docente da área de Endodontia da FOP-Unicamp.

Doutora em Clínica Odontológica, área de Endodontia da FOP-Unicamp. Professora adjunta da área de Endodontia da FOP-Unicamp.
} 
quedas e práticas esportivas ${ }^{2}$. Essas injúrias ocorrem com alta frequência em pré-escolares, crianças em idade escolar e adultos jovens, compreendendo $5 \%$ de todas as injúrias para as quais ocorre a procura pelo tratamento ${ }^{3-5}$.

Em relação à intervenção clínica, o trauma dentário é uma das situações de urgência odontológica mais desafiadoras, pois exige o diagnóstico preciso e o tratamento adequado para minimizar as complicações indesejáveis e atingir taxas de sobrevivência favoráveis para os dentes afetados ${ }^{6}$. As injúrias traumáticas podem causar alterações funcionais e estéticas, como fraturas, descoloração, dor e mobilidade, assim como afetar a fala, o sorriso e a autoconfiança ${ }^{7-9}$.

Dados sobre estudos epidemiológicos de traumatismos dentários são relatados em diversos países, sendo que a maioria desses é coletada de serviços públicos de saúde, embora alguns sejam advindos de serviços odontológicos em universidades, apontando que as injúrias traumáticas têm uma prevalência de $58,6 \%$ na dentição permanente e $36,8 \%$ na dentição decídua ${ }^{9-11}$.

Tendo em vista o exposto, o objetivo do presente estudo foi avaliar as características dos atendimentos realizados no que diz respeito ao gênero, à faixa etária, à etiologia, ao elemento dentário afetado, ao tempo decorrido entre o trauma e o primeiro atendimento, ao procedimento efetuado e à classificação da injúria dentária traumática dos pacientes atendidos no Serviço de Atendimento aos Traumatismos Dentários da Faculdade de Odontologia de Piracicaba da Universidade Estadual de Campinas (FOP-Unicamp), durante o período de dois anos.

\section{Sujeitos e método}

Este estudo foi aprovado pelo Comitê de Ética em Pesquisa com Seres Humanos da FOP-Unicamp, sob o protocolo de n⿳0 035/2013.

Realizou-se um estudo prospectivo, durante o período de fevereiro de 2013 a fevereiro de 2015 (Figura 1). Os dados referentes à identificação e à história médica e dental foram anotados na ficha clínica dos pacientes e os seguintes aspectos foram analisados: gênero, faixa etária, número de dentes acometidos, arcada e classificação do dente permanente traumatizado, tempo entre o trauma e o primeiro atendimento no serviço, etiologia do trauma, classificação da lesão dentária traumática e estrutura afetada do dente (estrutura dentária, envolvendo os tecidos duros do dente e/ou estrutura de suporte, envolvendo os tecidos periodontais).

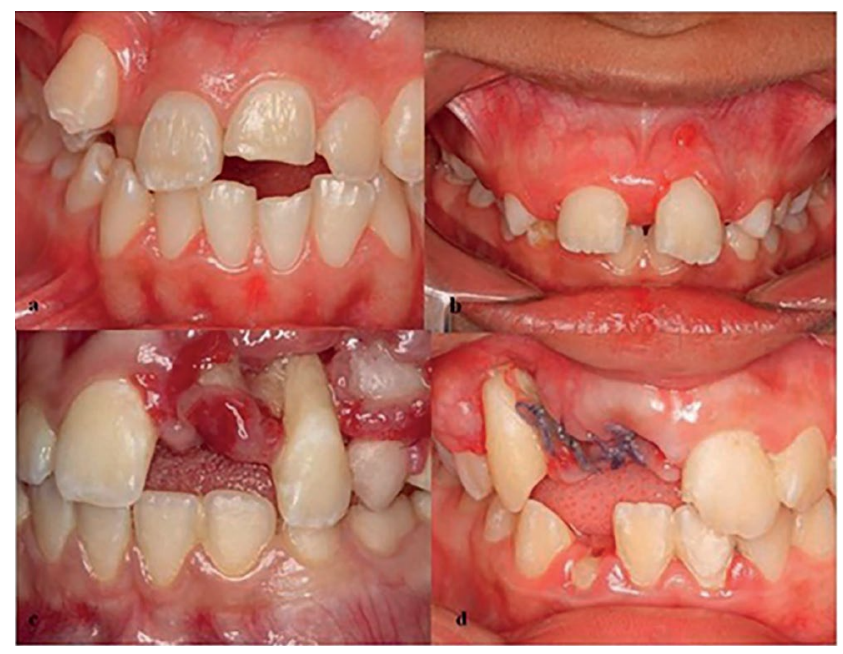

Figura 1 - Exemplos de traumas dentários: a) Fratura de esmalte e dentina do dente 21 ; b) Luxação extrusiva do dente 21; c) Luxação lateral do dente 22 e avulsão do dente 21; d) Avulsão dos dentes 11 e 12

Fonte: dados da pesquisa.

As causas dos traumatismos dentários foram divididas em: queda, acidentes com bicicleta, acidentes com motocicleta, acidentes de carro, acidentes de trabalho, atropelamento, esportes, agressão física e outros (causas que não se enquadram nas anteriores). O tipo de procedimento realizado foi dividido em: contenção semirrígida, exames clínico e radiográfico (testes de sensibilidade pulpar, testes de dor por percussão e palpação, presença de mobilidade, abscesso ou fístula e radiografia periapical), tratamento endodôntico, inserção da pasta obturadora preconizada no serviço, composta por hidróxido de cálcio, clorexidina $2 \%$ gel e óxido de zinco, restauração com resina composta, revascularização e outras opções que não se enquadram nas anteriores. A classificação da lesão dentária traumática foi baseada nas diretrizes da $\operatorname{IADT}^{5,12}$ e foi dividida em:

a) injúrias aos tecidos dentários: trinca de esmalte, fratura de esmalte, fratura de esmalte e dentina, fratura de esmalte, dentina e polpa, fratura coronorradicular e fratura radicular;

b) injúrias aos tecidos de suporte: concussão, subluxação, luxação extrusiva, luxação intrusiva, luxação lateral, avulsão e reimplante.

As informações coletadas foram organizadas em uma planilha Excel (Microsoft, Seattle, WA, EUA). A análise dos resultados foi calculada por meio do sistema SAS (SAS Institute Inc. The SAS System, release 9.3, Cary: NC, 2010). O teste do Qui-Quadrado foi utilizado para verificar a presença ou a ausência de significância. O nível de significância adotado foi de $5 \%$.

\section{Resultados}

Durante o período de fevereiro de 2013 a fevereiro de 2015, 57 pacientes foram atendidos no Serviço de Traumatismos Dentários da FOP-Unicamp, com o total de 161 dentes afetados. 
A Tabela 1 evidencia o gênero, a faixa etária, a arcada, os dentes mais afetados e o tempo entre o trauma e o primeiro atendimento no serviço, sendo que o gênero masculino foi o mais afetado $(39 / 68,4 \%)$, a faixa etária mais prevalente foi de pacientes abaixo dos 14 anos de idade (30/52,6\%). Os dentes mais acometidos foram: a arcada superior (140/87\%) e os dentes incisivos centrais (98/60,9\%). A maioria dos pacientes $(32 / 56,1 \%)$ procurou atendimento no serviço em torno de 0 a 30 dias após a ocorrência da injúria traumática.

Tabela 1 - Gênero, faixa etária, arcada, dentes mais afetados e tempo entre o trauma e o primeiro atendimento no serviço

\begin{tabular}{|c|c|c|c|}
\hline Características & Frequência & $\begin{array}{c}\text { Porcentagem } \\
(\%)\end{array}$ & $\begin{array}{c}\text { Valor de } \\
\mathrm{P}^{*}\end{array}$ \\
\hline \multicolumn{4}{|l|}{ Gênero } \\
\hline Feminino & 18 & 31,6 & 0.0020 \\
\hline Masculino & 39 & 68,4 & \\
\hline \multicolumn{4}{|l|}{ Faixa etária } \\
\hline$<14$ & 30 & 52,6 & \\
\hline $15-25$ & 15 & 26,3 & 0.0035 \\
\hline$>16$ & 12 & 21,1 & \\
\hline \multicolumn{4}{|l|}{ Arcada } \\
\hline Superior & 140 & 87,0 & $<0.001$ \\
\hline Inferior & 21 & 13,0 & \\
\hline \multicolumn{4}{|l|}{ Dente } \\
\hline IC & 98 & 60,9 & \\
\hline $\mathrm{IL}$ & 44 & 27,3 & $<0.001$ \\
\hline C & 8 & 5,0 & \\
\hline PM & 11 & 6,8 & \\
\hline \multicolumn{4}{|l|}{$\begin{array}{l}\text { Tempo do trauma } \\
\text { até o primeiro } \\
\text { atendimento }\end{array}$} \\
\hline 0-30 dias & 32 & 56,1 & \\
\hline 31-180 dias & 11 & 19,3 & 0.0022 \\
\hline 181-365 dias & 3 & 5,3 & \\
\hline$>365$ dias & 11 & 19,3 & \\
\hline
\end{tabular}

Fonte: dados da pesquisa.

A Tabela 2 mostra a etiologia do trauma e o número de dentes envolvidos na injúria traumática. As quedas (19/33,3\%) foram as causas mais frequentes das injúrias traumáticas e a maior parte dos pacientes contou com dois dentes envolvidos pelo trauma $(20 / 35,1 \%)$.
Tabela 2 - Etiologia do trauma e número de dentes envolvidos

\begin{tabular}{|c|c|c|c|}
\hline Características & Frequência & $\begin{array}{c}\text { Porcentagem } \\
(\%)\end{array}$ & $\begin{array}{l}\text { Valor } \\
\text { de P* }\end{array}$ \\
\hline \multicolumn{4}{|l|}{ Etiologia do trauma } \\
\hline Queda & 19 & 33,3 & \\
\hline Bicicleta & 12 & 21,1 & \\
\hline Atropelamento & 7 & 12,2 & \\
\hline Prática de esporte & 4 & 7,0 & \\
\hline Motocicleta & 4 & 7,0 & \\
\hline Acidente de carro & 3 & 5,3 & $<0.001$ \\
\hline Acidente de trabalho & 3 & 5,3 & \\
\hline Agressão física & 3 & 5,3 & \\
\hline Outras causas & 2 & 3,5 & \\
\hline \multicolumn{4}{|l|}{ Dentes envolvidos } \\
\hline 1 & 11 & 19,3 & \\
\hline 2 & 20 & 35,1 & \\
\hline 3 & 8 & 14,0 & \\
\hline 4 ou mais & 18 & 31,6 & $<0.001$ \\
\hline
\end{tabular}

A Tabela 3 descreve a estrutura afetada e o tipo de atendimento realizado. Injúrias envolvendo as estruturas de suporte $(80 / 49,7 \%)$ foram as mais frequentes. $\mathrm{O}$ procedimento mais efetuado foi a realização dos exames clínico e radiográfico $(75 / 46,6 \%)$, seguido do tratamento com a inserção da pasta obturadora preconizada pelo serviço, composta por hidróxido de cálcio, clorexidina $2 \%$ gel e óxido de zinco $(27 / 16,8 \%)$.

Tabela 3 - Estrutura afetada e tratamento realizado

\begin{tabular}{|c|c|c|c|}
\hline Características & Frequência & $\begin{array}{c}\text { Porcentagem } \\
(\%)\end{array}$ & $\begin{array}{l}\text { Valor de } \\
\qquad \mathrm{P}^{*}\end{array}$ \\
\hline \multicolumn{4}{|l|}{ Estrutura afetada } \\
\hline Dentária & 48 & 29,8 & \multirow{4}{*}{0.0013} \\
\hline Suporte & 80 & 49,7 & \\
\hline $\begin{array}{l}\text { Combinada (dentária } \\
+ \text { suporte) }\end{array}$ & 33 & 20,5 & \\
\hline \multicolumn{3}{|l|}{ Tratamento realizado } & \\
\hline $\begin{array}{l}\text { Exames clínico e } \\
\text { radiográfico }\end{array}$ & 75 & 46,6 & \\
\hline Pasta obturadora & 27 & 16,8 & \\
\hline $\begin{array}{l}\text { Tratamento } \\
\text { endodôntico }\end{array}$ & 26 & 16,1 & \\
\hline $\begin{array}{l}\text { Restauração com } \\
\text { resina composta }\end{array}$ & 10 & 6,2 & $<0.001$ \\
\hline Contenção semirrígida & 13 & 8,1 & \\
\hline Revascularização & 5 & 3,1 & \\
\hline Outros & 5 & 3,1 & \\
\hline
\end{tabular}

*Teste do Qui-Quadrado

Fonte: dados da pesquisa 
A Figura 2 mostra que a fratura coronária de esmalte e dentina $(32 / 19,9 \%, \mathrm{p}<0.001$, teste do Qui-Quadrado) apresentou-se como a injúria mais prevalente aos tecidos duros dentários, seguida de fratura de esmalte, dentina e polpa (21/13\%) e fratura coronária de esmalte (15/9,3\%). A Figura 3 evidencia que a subluxação $(31 / 19,3 \%, \mathrm{p}<0.001$, teste do Qui-Quadrado) apresentou-se como a injúria mais comum aos tecidos de suporte, seguida de avulsão $(26 / 16,1 \%)$ e luxação lateral $(20 / 12,4 \%)$.

\section{Injúrias aos tecidos dentários}

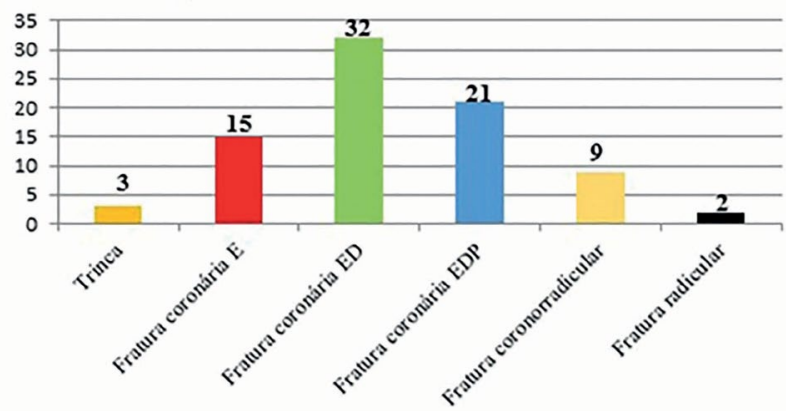

Figura 2 - Casuística das injúrias aos tecidos duros dos dentes traumatizados

Legenda: Fratura coronária E: fratura coronária de esmalte; Fratura coronária ED: fratura coronária de esmalte e dentina; Fratura coronária EDP: fratura coronária de esmalte, dentina e polpa.

Fonte: dados da pesquisa

Injúrias aos tecidos de suporte

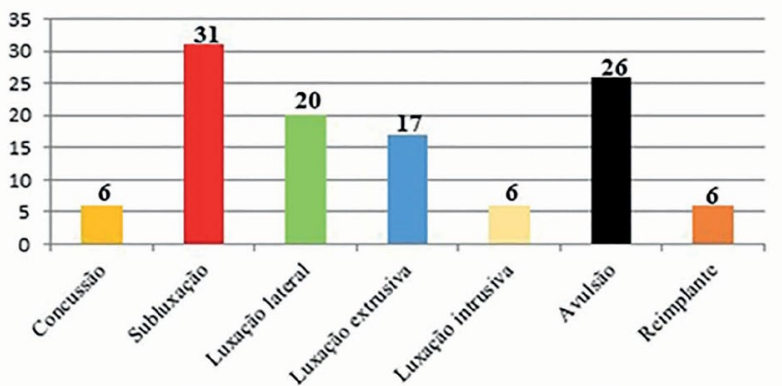

Figura 3 - Casuística das injúrias aos tecidos de suporte dos dentes traumatizados

Fonte: dados da pesquisa.

\section{Discussão}

O trauma dental é uma das injúrias mais comuns durante a infância e a adolescência, com taxas de prevalência semelhantes à cárie dentária e à doença periodontal ${ }^{13}$. Portanto, estudos que verifiquem sua incidência em clínicas, serviços públicos e faculdades são importantes para que possam ser feitos o diagnóstico e o tratamento corretos, assim como campanhas educativas, no intuito de orientar a prevenção e apontar o que fazer nos casos em que ocorrem as injúrias dentais traumáticas.

$\mathrm{Na}$ presente pesquisa, o gênero masculino foi o mais afetado pelos traumatismos dentários, o que está de acordo com estudos prévios ${ }^{6,13-19}$. Essa cons- tatação pode ser atribuída ao fato de os homens serem mais hiperativos e se envolverem mais frequentemente em atividades físicas, como esportes de contato, geralmente sem o uso de proteção adequada, e, também, em jogos mais agressivos como as lutas ${ }^{16,19}$. A faixa etária mais prevalente foi de crianças abaixo dos 14 anos de idade, o que foi, igualmente, encontrado nos estudos de Bücher et al. ${ }^{6}$, Toprak et al. ${ }^{13}$ e Soares et al. ${ }^{19}$, enquanto Souza-Filho et al. ${ }^{14}$ verificaram que indivíduos de 15 a 25 anos de idade foram os mais acometidos por traumatismos dentários.

$\mathrm{O}$ incisivo central superior foi o dente mais afetado, sendo esse dado unânime em muitos estu$\operatorname{dos}^{6,13,15-19}$. Isso se deve à sua posição vulnerável na cavidade bucal, podendo, também, estar evolvido com selamento labial inadequado e overjet acentuado, o que torna o elemento dentário ainda mais predisponente a uma injúria traumática ${ }^{16,20}$.

A maioria dos pacientes compareceu ao serviço dentro dos primeiros 30 dias de ocorrência do trauma, enquanto que Bücher et al. ${ }^{6}$ relataram que a maior parte dos pacientes compareceu à consulta nas primeiras 36 horas após o acidente. O tempo decorrido entre o trauma e o primeiro atendimento no serviço encontrado no estudo é relativamente baixo. Isso demonstra que os pacientes e responsáveis estão razoavelmente bem informados sobre o que fazer nos casos de trauma dentário, visto que quanto menor o tempo entre o trauma e o primeiro atendimento, maior a chance de sobrevivência do elemento dentário. O serviço da FOP-Unicamp é mais caracterizado por receber pacientes que já foram submetidos ao atendimento imediato e foram encaminhados para dar continuidade ao tratamento, geralmente por hospitais ou postos de saúde da região.

As quedas foram a etiologia mais comum das injúrias traumáticas, seguidas por acidentes de bicicleta, o que também foi encontrado em outros estudos $^{6,13-16,19}$. Crianças menores de 14 anos de idade são mais acometidas por traumatismos dentários durante brincadeiras em casa ou na escola ou passeios de bicicleta, enquanto que os jovens estão mais sujeitos a acidentes de trânsito ou praticam mais esportes. Nesses casos, o uso de protetor bucal pode ser de grande utilidade para prevenir acidentes $^{13,14}$.

A maior parte dos pacientes apresentou dois ou mais dentes envolvidos pelo trauma, corroborando com os achados de Guedes et al. ${ }^{16}$, enquanto Freire et $a .^{18}$ encontraram que a maioria dos traumatismos contou com apenas um dente acometido.

$\mathrm{O}$ procedimento mais frequente no serviço foi a realização dos exames clínico e radiográfico, o que está de acordo com os resultados de Toprak et al. ${ }^{13}$ e difere do estudo de Chen et al. ${ }^{17}$, que relataram o tratamento endodôntico como o mais efetuado. Isso se deve ao fato de que a maioria das lesões foi de subluxação, em que, muitas vezes, apenas 
os exames clínico e radiográfico são suficientes, seguidos de consultas de acompanhamento. Além disso, alguns dos pacientes receberam atendimento de urgência em hospitais ou postos de saúde e, em seguida, foram encaminhados ao serviço para a continuidade do tratamento. $\mathrm{O}$ segundo procedimento mais frequente foi o tratamento realizado com a pasta obturadora preconizada pelo serviço, composta por hidróxido de cálcio, clorexidina $2 \%$ gel e óxido de zinco, utilizado em casos de ápices incompletamente formados e em traumas mais severos, como luxações e reimplantes ${ }^{21-23}$.

Apesar dos diferentes critérios de classificação encontrados na literatura, as fraturas coronárias de esmalte e dentina foram as injúrias traumáticas mais prevalentes envolvendo os tecidos duros, o que está de acordo com estudos anteriores ${ }^{6,13-16,19,20}$. Em relação às injúrias aos tecidos de suporte, a subluxação e a avulsão foram as mais comuns, o que também pode ser encontrado em estudos prévios ${ }^{6,19}$.

No presente estudo, foi verificado que, dos 32 dentes avulsionados, apenas seis foram reimplantados, enquanto que Zhang e Gong ${ }^{24}$ relataram que, de 120 dentes avulsionados, 85 foram reimplantados. Os dentes avulsionados devem ser reimplantados assim que possível e, caso não sejam, devem ser armazenados em meios adequados, como leite ou soro fisiológico, e o paciente deve procurar um serviço odontológico o mais rápido possível. Os fatores mais críticos para o sucesso do reimplante são o meio de transporte e o tempo em que o dente ficou fora da cavidade bucal ${ }^{24,25}$. Os dados encontrados neste estudo sugerem a necessidade de realização de campanhas educativas informando quais os meios de transporte mais adequados para um dente avulsionado e a conscientização da necessidade do atendimento imediato.

Os resultados da casuística encontrada no Serviço de Atendimento aos Traumatismos Dentários da FOP-Unicamp durante o período de dois anos analisado são semelhantes aos encontrados em trabalhos realizados tanto no Brasil quanto em outros países. Isso caracteriza a importância desses estudos no sentido de ajudar na adoção de campanhas educativas e preventivas sobre as injúrias traumáticas, assim como a necessidade de oferecer um serviço que atenda a demanda dos casos de traumatismos dentários, possibilitando a efetivação de diagnóstico, tratamento e acompanhamento adequados, visando minimizar as possíveis sequelas resultantes desses acidentes $^{16}$.

\section{Conclusões}

Concluiu-se que, durante o período de dois anos analisado, os achados mais prevalentes dos atendimentos realizados no Serviço de Traumatismos Dentários da FOP-Unicamp foram: o gênero masculino, a maior frequência de injúrias dentárias traumáticas ocorrida na faixa etária abaixo dos 14 anos de idade; os incisivos centrais como os dentes mais acometidos; o período de tempo entre 0 a 30 dias para procurar atendimento no serviço; as quedas como a etiologia mais comum; o exame clínico e radiográfico como o procedimento mais efetuado e as fraturas coronárias de esmalte e dentina e a subluxação como as injúrias mais observadas.

\section{Abstract}

Objective: To verify the frequency of care provided at the Dental Trauma Service of the School of Dentistry Piracicaba in the University of Campinas, SP, Brazil (FOP-UNICAMP), from February 2013 to February 2015. Subjects and methods: Data were collected from clinical records used in the service, and the following aspects were assessed: gender, age group, teeth classification, time between trauma and the first care visit, etiology, classification of traumatic dental injury, and procedure performed. Data were analyzed aided by the SAS software and the chi-square test used to verify the presence or absence of significance at level of 5\%. Results: Clinical records from 57 patients with 161 traumatized teeth were analyzed; there was higher prevalence of the male gender and age group under 14 years. Central incisors $(60.9 \%)$ were the most affected teeth. Most patients (56.1\%) sought care from 0 to 30 days after trauma. Falls (33.3\%) were the most common etiology. The most frequent traumatic dental injuries were crown fractures of enamel and dentin (19.9\%) and subluxation (19.3\%). Clinical and radiographic examinations $(46.6 \%)$ were the most performed procedures. Conclusion: The most prevalent findings of care visits were male gender, highest frequency of injuries in age group under 14 years, central incisors as the most affected teeth, period between 0-30 days to seek care, falls as the most common etiology, clinical and radiographic examinations as most performed procedures, and crown fractures of enamel and dentin and subluxation as the most observed injuries.

Keywords: Endodontics. Epidemiology. Dental traumas.

\section{Referências}

1. Glendor U. Epidemiology of traumatic dental injuries a 12 year review of the literature. Dent Traumatol 2008; 24(6):603-11.

2. Santos SE, Marchiori EC, Soares AJ, Asprino L, Souza Filho FJ, Moraes M, et al. A 9-year retrospective study of dental trauma in Piracicaba and neighboring regions in the state of São Paulo, Brazil. J Oral Maxillofac Surg 2010; 68(8):1826-32.

3. Petersson EE, Andersson L, Sorensen S. Traumatic oral vs non-oral injuries. Swed Dent J 1997; 21(1-2):55-68.

4. Andreasen JO, Andreasen FM. Classificação, etiologia e epidemiologia. In: Andreasen JO, Andreasen FM, editores. Texto e atlas colorido de traumatismo dental. 3. ed. São Paulo: Artmed; 2001. p. 151-80.

5. DiAngelis J, Andreasen JO, Ebeleseder A, Kenny DJ, Trope M, Sigurdsson A, et al. International association of dental traumatology guidelines for the management of traumatic 
dental injuries: 1 . Fractures and luxations of permanent teeth. Dent Traumatol 2012; 28(1):2-12.

6. Bücher K, Neumann C, Hickel R, Kühnisch J. Traumatic dental injuries at a German University Clinic 2004-2008. Dent Traumatol 2013; 29(2):127-33.

7. Bendo CB, Paiva SM, Oliveira AC, Goursand D, Torres CS, Pordeus IA, et al. Prevalence and associated factors of traumatic dental injuries in Brazilian schoolchildren. J Public Health Dent 2010; 70(4):313-8.

8. Ramos-Jorge J, Paiva SM, Tataounoff J, Pordeus IA, Marques LS, Ramos-Jorge ML. Impact of treated/untreated traumatic dental injuries on quality of life among Brazilian schoolchildren. Dent Traumatol 2014; 30(1):27-31.

9. Rajab LD. Traumatic dental injuries in children presenting for treatment at the Department of Pediatric Dentistry, Faculty of Dentistry, University of Jordan, 1997-2000. Dent Traumatol 2003; 19(1):6-11.

10. Rezende FMCR, Gaujac C, Rocha AC, Peres MPSM. A prospective study of dentoalveolar trauma at the Hospital das Clínicas, São Paulo University Medical School. Clinics 2007; 62(2):133-8.

11. Ekanayake L, Perera M. Pattern of traumatic dental injuries in children attending the University Dental Hospital: Sri Lanka. Dent Traumatol 2008; 24(4):471-4.

12. Andersson L, Andreasen JO, Day P, Heithersay G, Trope M, DiAngelis AJ, et al. International association of dental traumatology guidelines for the management of traumatic dental injuries: 2. Avulsion of permanent teeth. Dent Traumatol 2012; 28(2):88-96.

13. Toprak ME, Tuna EB, Seymen F, Gençay K. Traumatic dental injuries in Turkish children, Istanbul. Dent Traumatol $2014 ; 30(4): 280-4$

14. Souza-Filho FJ, Soares AJ, Gomes BPFA, Zaia AA, Ferraz CCR, Almeida JFA. Avaliação das injúrias traumáticas observadas no Centro de Trauma Dental da Faculdade de Odontologia de Piracicaba - Unicamp. RFO UPF 2009; 14(2):111-6.

15. Díaz JA, Bustos L, Brandt AC, Fernández BE. Dental injuries among children and adolescents aged 1-15 years attending to public hospital in Temuco, Chile. Dent Traumatol 2010; 26(3):254-61.

16. Guedes OA, Alencar AHG, Lopes LG, Pécora JD, Estrela C. A retrospective study of traumatic dental injuries in a Brazilian Dental Urgency Service. Braz Dent J 2010; 21(2):153-7.

17. Chen Z, Si Y, Gong Y, Wang JG, Liu JX, He Y, et al. Traumatic dental injuries among 8-to 12-year-old schoolchildren in Pinggu District, Beijing, China, during 2012. Dent Traumatol 2014; 30(5):385-90

18. Freire MC, Vasconcelos DN, Vieira AS, Araújo JA, Moreira RS, Nunes MF. Association of traumatic dental injuries with individual-, sociodemographic- and school- related factores among schoolchildren in Midwest Brazil. Int J Environ Res Public Health 2014; 11(9):9885-96.

19. Soares TRC, Risso PA, Maia LC. Traumatic dental injury in permanent teeth of young patients attended at the federal University of Rio de Janeiro, Brazil. Dent Traumatol 2014; 30(4):312-6.

20. Schatz JP, Hakeberg M, Ostini E, Kiliaridis S. Prevalence of traumatic injuries to permanent dentition and its association with overjet in a Swiss child population. Dent Traumatol 2013; 29(2):110-4.

21. Soares AJ, Lima TFR, Lins FF, Herrera DR, Gomes BPFA, Souza-Filho FJ. Um nuevo protocolo de medicación intraconducto para dientes com necrosis pulpar y rizogénesis incompleta. Rev Estomatol Herediana 2011; 21(3):145-9.
22. Soares AJ, Nagata JY, Casarin RCV, Almeida JFA, Gomes BPFA, Zaia AA, et al. Apexification with a new intra-canal medicament: a multidisciplinary case report. Iran Endod J 2012; 7(3):165-70

23. Soares AJ, Lima TFR, Nagata JY, Gomes BPFA, Zaia AA, Souza-Filho FJ. Intracanal dressing paste composed by calcium hydroxide, chlorhexidine and zinc oxide for the treatment of immature and mature traumatized teeth. Braz J Oral Sci 2014; 13(1):6-11.

24. Zhang X, Gong Y. Characteristics of avulsed permanent teeth treated at Beijing Stomatological Hospital. Dent Traumatol 2011; 27(5):379-84.

25. Soares AJ, Gomes BPFA, Zaia AA, Ferraz CCR, Souza-Filho FJ. Relationship between clinical radiographic evaluation and outcome of teeth replantation. Dental Traumatol 2008; 24(2):183-8

\section{Endereço para correspondência:}

Adriana de Jesus Soares

Avenida Limeira, 901

Areião

13414-903 Piracicaba, SP

Telefone: (19) 2106-5215

E-mail: ajsoares.endo@uol.com.br

Recebido: 17/07/2015. Aceito: 22/04/2016. 